Research paper

\title{
Quantifying covalent interactions with resonant inelastic soft X-ray scattering: Case study of $\mathrm{Ni}^{2+}$ aqua complex
}

\author{
K. Kunnus ${ }^{\mathrm{a}, \mathrm{b}, *}$, I. Josefsson ${ }^{c}$, S. Schreck ${ }^{\mathrm{a}, \mathrm{b}}$, W. Quevedo ${ }^{\mathrm{a}}$, P.S. Miedema ${ }^{\mathrm{a}}$, S. Techert ${ }^{\mathrm{d}, \mathrm{e}}$, F.M.F. de Groot $^{\mathrm{f}}$, \\ A. Föhlisch ${ }^{\mathrm{a}, \mathrm{b}}$, M. Odelius ${ }^{\mathrm{c}}$, Ph. Wernet $^{\mathrm{a}}$ \\ a Institute for Methods and Instrumentation for Synchrotron Radiation Research, Helmholtz-Zentrum Berlin für Materialien und Energie GmbH, 12489 Berlin, Germany \\ ${ }^{\mathrm{b}}$ Institut für Physik und Astronomie, Universität Potsdam, Karl-Liebknecht-Strasse 24/5, 1476 Potsdam, Germany \\ ${ }^{\mathrm{c}}$ Department of Physics, Stockholm University, AlbaNova University Center, 10691 Stockholm, Sweden \\ ${ }^{\mathrm{d}}$ Max Planck Institute for Biophysical Chemistry, Am Faßberg 11, 37077 Göttingen, Germany \\ e Structural Dynamics of (Bio)chemical Systems, DESY, Notkestrasse 85, 22607 Hamburg, Germany \\ ${ }^{\mathrm{f}}$ Department of Chemistry, Utrecht University, Universiteitsweg 99, 3584 CG Utrecht, Netherlands
}

\section{A R T I C L E I N F O}

\section{Article history:}

Received 21 September 2016

In final form 20 December 2016

Available online 23 December 2016

\section{Keywords:}

Transition-metal ion

Aqueous solution

Covalent interaction

Resonant inelastic X-ray scattering

Ligand-field state

Charge-transfer state

\begin{abstract}
A B S T R A C T
We analyze the effects of covalent interactions in Ni 2p3d resonant inelastic X-ray scattering (RIXS) spectra from aqueous $\mathrm{Ni}^{2+}$ ions and find that the relative RIXS intensities of ligand-to-metal charge-transfer final states with respect to the ligand-field final states reflect the covalent mixing between $\mathrm{Ni} 3 \mathrm{~d}$ and water orbitals. Specifically, the experimental intensity ratio at the $\mathrm{Ni}_{2}$-edge allows to determine that the Ni $3 \mathrm{~d}$ orbitals have on average $5.5 \%$ of water character. We propose that $2 \mathrm{p} 3 \mathrm{~d}$ RIXS at the Ni $\mathrm{L}_{3}{ }^{-}$ edge can be utilized to quantify covalency in $\mathrm{Ni}$ complexes without the use of external references or simulations.
\end{abstract}

(c) 2016 Elsevier B.V. All rights reserved.

\section{Introduction}

Covalency, understood as the sharing of electrons between atomic centers [1], is a fundamental concept that is essential for understanding and predicting basic chemical properties ranging from molecular structures to reactivity and biological function. However, direct experimental determination of covalency, i.e. contributions of atomic orbitals from different atomic centers to a molecular orbital (MO), is a generally a difficult task. Here we demonstrate how resonant inelastic X-ray scattering (RIXS) at a 3d transition metal L-edge can be employed to experimentally derive quantitative information on covalent interactions by revealing the atomic compositions of the chemically relevant frontier orbitals. We test our concept by deriving the covalent contributions to the solute-solvent interactions of $\mathrm{Ni}^{2+}$ ions in aqueous solution. The interactions of $\mathrm{Ni}^{2+}$ with the solvent water molecules can be well described by considering the first solvation shell only [2] with octahedral arrangement of the water ligands. The highly polar coordinative bonding between $\mathrm{Ni}$ and the water ligands in

\footnotetext{
* Corresponding author. Current address: PULSE Institute, SLAC National Accelerator Laboratory, Menlo Park, CA 94025, USA.

E-mail address: kkunnus@stanford.edu (K. Kunnus).
}

the resulting prototypical Werner-type $\left[\mathrm{Ni}\left(\mathrm{H}_{2} \mathrm{O}\right)_{6}\right]^{2+}$ complex is characterized by weak covalent interactions of the ligand-tometal charge-transfer type ideally serving for a case study [3-5].

Recently, triggered by the development of soft X-ray techniques on liquids and solutions, a number of X-ray absorption spectroscopy (XAS), photoelectron and Auger electron spectroscopy studies have addressed 3d-transition-metal ions in water [2,612]. Given the intrinsic sensitivity to local charge distributions, these studies emphasized the covalent effects in metal-solute bonding. This sensitivity of X-ray spectroscopy methods to ligand environment is frequently utilized not only by soft X-ray, but also by hard X-ray techniques [13-18]. However, quantitative determination of the orbital specific covalent contributions is in practice often beyond the experimental capabilities. Particularly investigations at the transition metal absorption edges require typically elaborate electronic structure calculations and spectral simulations in order to disentangle the inter-atomic covalent effects from intra-atomic and core-hole relaxation effects [8,19-24], although significant advancements with regard to density functional and wavefunction based computational methods have been made in recent years [25-29].

In contrast to sophisticated computational methods, a relatively simple method to derive quantitative information about the 
metal-ligand covalency from more experimental grounds has been developed by Solomon and co-workers [30]. They derived a formula based on the molecular orbital theory to describe the intensity of ligand K-edge XAS pre-edge feature:

$I_{X A S} \propto \alpha^{2} n_{h}(1-k)^{2}\left|\left\langle\phi_{L 1 S}|\hat{D}| \phi_{\text {Lnp }}\right\rangle\right|^{2}$

Here $\alpha^{2}$ is metal-ligand covalency (defined as the ligand content in the MO corresponding to the pre-edge feature), $\mathrm{n}_{\mathrm{h}}$ is number of holes in the respective MO manifold, $\mathrm{k}$ describes the contribution of the other ligand atoms to the MO and the last term is a square of the atomic transition dipole moment. The aim of this letter is to derive an analogues expression for metal 2p3d RIXS in order to fully exploit the information content embedded in the RIXS spectral intensities.

In our previous experimental work we analyzed in detail the metal-centered ligand-field (LF) RIXS final states at the $\mathrm{Ni}_{3}$ and $\mathrm{L}_{2}$ edge [31]. Crystal field multiplet (CFM) and restricted active space self-consistent field (RASSCF) calculations presented there did not include ligand-to-metal charge-transfer (LMCT) final states due to computational limitations. In contrast, the present theoretical study concentrates on the LMCT peaks. Our objective is to relate the relative RIXS intensity of LF and LMCT peak to the degree of covalency of the $\mathrm{Ni}^{2+}$-water bond, apply it to the experimental spectrum from Ref. [31] and carry out additional charge transfer multiplet (CTM) calculations which include both LF and LMCT final states.

\section{Theory}

RIXS spectra were simulated by multiplying absorption and emission transition moments (two-step approximation) as retrieved from the CTM calculations [32-34]. Exact diagonalization was utilized to calculate the valence- and core-excited states [35]. The following convolution scheme was applied (values for the FWHM are given): $0.35 \mathrm{eV}$ Gaussian broadening taking into account the monochromator bandwidth, $0.5 \mathrm{eV}(1.0 \mathrm{eV})$ Lorentzian lifetime broadening at the $\mathrm{L}_{3}\left(\mathrm{~L}_{2}\right)$ edge for core-excited states, and $1.2 \mathrm{eV}$ Gaussian broadening of RIXS spectra to account for the spectrometer resolution. An additional $0.5 \mathrm{eV}$ Gaussian broadening of both X-ray absorption and RIXS spectra was used to account for inhomogeneous broadening. Polarization effects were not included in the simulation of the RIXS spectra (in Ref. [31] we found these are small in horizontal polarization).

\section{Results and discussion}

Water $3 \mathrm{a}_{1}\left(\mathrm{a}_{1 \mathrm{~g}}+\mathrm{e}_{\mathrm{g}}+\mathrm{t}_{1 \mathrm{u}}\right)$ orbitals are involved in $\sigma$-bonding with the Ni $3 d\left(e_{g}\right), 4 s\left(a_{1 g}\right)$ and $4 \mathrm{p}\left(\mathrm{t}_{1 \mathrm{u}}\right)$ orbitals whereas water $1 \mathrm{~b}_{1}\left(\mathrm{t}_{2 \mathrm{~g}}+-\right.$ $\left.t_{2 u}\right)$ and $\mathrm{Ni} 3 d\left(t_{2 g}\right)$ orbitals exhibit $\pi$-interactions in $\left[\mathrm{Ni}\left(\mathrm{H}_{2} \mathrm{O}\right)_{6}\right]^{2+}$ $[4,5]$. The leading covalent contribution has been identified as the mixing between Ni $3 d$ and the water $3 a_{1}$ and $1 b_{1}$ orbitals $[5,36]$. The most relevant MOs for the present investigation are the bonding $\left(e_{g}, t_{2 g}\right)$ nominal ligand and antibonding $\left(e_{g}^{*}, t_{2 g}^{*}\right)$ nominal $\mathrm{Ni}$ MOs as displayed in Fig. 1(a).

The principle of utilizing L-edge RIXS for probing covalency in $3 \mathrm{~d}$ transition-metal complexes is illustrated in Fig. 1(b) and (c). Resonant excitation to unoccupied nominal $\mathrm{Ni} 3 \mathrm{~d}$ MOs at the $\mathrm{Ni}$ L-edge is followed by scattering to the LF final states of the $\left(\mathrm{e}_{\mathrm{g}}^{*}\right.$, $\left.\mathrm{t}_{2 \mathrm{~g}}^{*}\right)^{8}$ configuration or by scattering to the LMCT final states of the $\left(\mathrm{e}_{\mathrm{g}}, \mathrm{t}_{2 \mathrm{~g}}\right)^{9}\left(\mathrm{e}_{\mathrm{g}}^{*}, \mathrm{t}_{2 \mathrm{~g}}^{*}\right)^{9}$ configuration (Fig. 1(b)) [31].

In first approximation the intensities of the LF and LMCT RIXS peaks reflect the $\mathrm{Ni} 3 \mathrm{~d}$ composition of the $\left(\mathrm{e}_{\mathrm{g}}^{*}, \mathrm{t}_{2 \mathrm{~g}}^{*}\right)$ and $\left(\mathrm{e}_{\mathrm{g}}, \mathrm{t}_{2 \mathrm{~g}}\right)$ MOs, respectively (Fig. 1(c)). Because these MOs are the antibonding and bonding combinations of $\mathrm{Ni} 3 \mathrm{~d}$ and water $3 \mathrm{a}_{1}$ and $1 \mathrm{~b}_{1}$ orbitals resulting from the corresponding covalent orbital mixings, the (a)

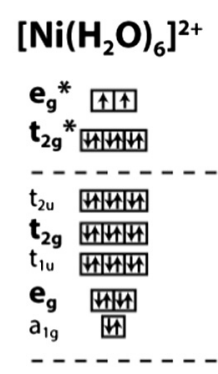

$2 p$ 田柴困 (b)

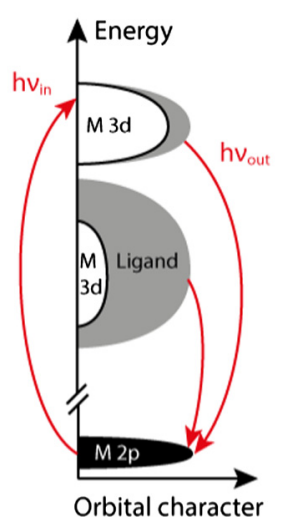

(c)

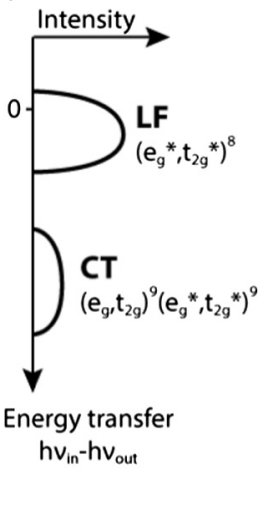

Fig. 1. (a) Schematic molecular orbital (MO) diagram for the hexaaqua $\left[\mathrm{Ni}\left(\mathrm{H}_{2} \mathrm{O}\right)_{6}\right]^{2+}$ complex. Bold letters are used for the orbitals relevant for the present investigation. $\mathrm{e}_{\mathrm{g}} / \mathrm{e}_{\mathrm{g}}^{*}$ and $\mathrm{t}_{2 \mathrm{~g}} / \mathrm{t}_{2 \mathrm{~g}}^{*}$ MOs form two sets of bonding/antibonding orbitals, governing the covalent interaction. (b) Schematic depiction of the resonant inelastic X-ray scattering (RIXS) process in a transition-metal complex. The metal $3 \mathrm{~d}$ and ligand characters of the MOs are shown in white and grey, respectively. For $\left[\mathrm{Ni}\left(\mathrm{H}_{2} \mathrm{O}\right)_{6}\right]^{2+}$ these are $\mathrm{Ni} 3 \mathrm{~d}$ and water $3 \mathrm{a}_{1} / 1 \mathrm{~b}_{1}$ characters in the nominal metal $\mathrm{e}_{\mathrm{g}}^{*}$ and $\mathrm{t}_{2 \mathrm{~g}}^{*}$ and nominal ligand $e_{g}$ and $t_{2 g}$ MOs. $h v_{\text {in }}$ and $h v_{\text {out }}$ denote the incident and the emitted photon energies. (c) Schematic RIXS spectrum with RIXS intensities versus energy transfer. The peak denoted LF corresponds to RIXS final states assigned to valenceexcited ligand-field states [states of the $\left(\mathrm{e}_{\mathrm{g}}^{*}, \mathrm{t}_{2 \mathrm{~g}}^{*}\right)^{8}$ configuration for $\mathrm{Ni}^{2+}(\mathrm{aq})$ ] and the peak denoted CT corresponds to RIXS final states assigned to ligand-to-metal charge-transfer states $\left[\left(\mathrm{e}_{\mathrm{g}}, \mathrm{t}_{2 \mathrm{~g}}\right)^{9}\left(\mathrm{e}_{\mathrm{g}}^{*}, \mathrm{t}_{2 \mathrm{~g}}^{*}\right)^{9}\right.$ configuration for $\left.\mathrm{Ni}^{2+}(\mathrm{aq})\right]$. The intensities of LF and CT peaks reflect the metal $3 d(\mathrm{Ni} 3 \mathrm{~d})$ character of the MOs $\left[\left(\mathrm{e}_{\mathrm{g}}^{*}, \mathrm{t}_{2 \mathrm{~g}}^{*}\right)\right.$ and $\left(\mathrm{e}_{\mathrm{g}}\right.$, $\left.\left.\mathrm{t}_{2 \mathrm{~g}}\right)\right]$.

relative intensities of LF and LMCT RIXS peaks can be expected to correlate with the magnitude of these mixings, i.e. the covalency in the complex.

The qualitative considerations outlined above are scrutinized as follows. Within the framework of LCAO-MO theory the covalent bonding between atoms is described in terms of mixing of atomic orbitals at different atomic sites. In case of metal-centered complexes such as $\left[\mathrm{Ni}\left(\mathrm{H}_{2} \mathrm{O}\right)_{6}\right]^{2+}$ we can describe the bonding between the metal and the ligands as a mixing of the symmetry adapted metal orbitals with ligand group orbitals (LGO) formed by a symmetry adapted mixing of the equivalent ligand orbitals. Mixing of one metal orbital with one LGO results in a pair of bonding and antibonding MOs:

$\psi=\alpha \phi_{M}+\sqrt{1-\alpha^{2}} \phi_{L}$

$\psi^{*}=\sqrt{1-\alpha^{2}} \phi_{M}-\alpha \phi_{L}$

where $\psi$ is a bonding and $\psi^{*}$ an antibonding $\mathrm{MO}, \phi_{M}$ is an atomic metal orbital and $\phi_{\mathrm{L}}$ is a LGO. The parameter $\alpha^{2}$ describes the magnitude of mixing between the metal orbital and the LGO and can be interpreted as the relative contribution of ligand orbitals to the antibonding MO (or the relative amount of metal orbitals in the bonding MO). We have followed here the same definition of covalency as used by Solomon et al. [19,30].

Within approximations detailed below (most importantly the one-electron and the frozen-orbital approximations), the dipole transition matrix elements involving decay from the $\psi$ and $\psi^{*}$ MOs to an atomic metal 2 p core orbital $\phi_{\mathrm{M} 2 \mathrm{p}}$ can be written as:

$$
\begin{aligned}
& \left|\left\langle\psi|\hat{D}| \phi_{M 2 p}\right\rangle\right|^{2}=\alpha^{2}\left|\left\langle\phi_{M 3 d}|\hat{D}| \phi_{M 2 p}\right\rangle\right|^{2} \\
& \left|\left\langle\psi^{*}|\hat{D}| \phi_{M 2 p}\right\rangle\right|^{2}=\left(1-\alpha^{2}\right)\left|\left\langle\phi_{M 3 d}|\hat{D}| \phi_{M 2 p}\right\rangle\right|^{2}
\end{aligned}
$$

where $\phi_{\mathrm{M} 3 \mathrm{~d}}$ is a metal $3 \mathrm{~d}$ orbital, $\phi_{\mathrm{M} 2 \mathrm{p}}$ is a metal $2 \mathrm{p}$ core orbital and $\mathrm{D}$ is the dipole transition operator. The above equations indicate that the transition probabilities are proportional to the $3 \mathrm{~d}$ character 
of the respective orbitals. To describe the relative RIXS intensities, the number and occupation of the $\psi$ and $\psi^{*}$ MOs in the coreexcited states has to be taken into account in addition. In case of $\left[\mathrm{Ni}\left(\mathrm{H}_{2} \mathrm{O}\right)_{6}\right]^{2+}$ we must consider the two sets of bonding and antibonding orbitals $\left(e_{g}, e_{g}^{*}\right)$ and $\left(t_{2 g}, t_{2 g}^{*}\right)$ with different covalency that we define as $\alpha_{\mathrm{Eg}}^{2}$ and $\alpha_{\mathrm{T} 2 \mathrm{~g}}^{2}$. The occupation of these orbitals in the core-excited states is evident from the electronic configurations of the core-excited states, namely $(2 p)^{5}\left(e_{g}\right)^{4}\left(t_{2 g}\right)^{6}\left(t_{2 g}^{*}\right)^{6}\left(e_{g}^{*}\right)^{3}$. The relative intensities of LF and LMCT RIXS peaks is thus

$\frac{I_{L M C T}}{I_{L F}}=\frac{4 \alpha_{E g}^{2}+6 \alpha_{T 2 g}^{2}}{3\left(1-\alpha_{E g}^{2}\right)+6\left(1-\alpha_{T 2 g}^{2}\right)}$

To experimentally determine the average covalency we introduce the weighted average $\left\langle\alpha^{2}\right\rangle=(4 / 10)^{*} \alpha_{\mathrm{Eg}}^{2}+(6 / 10)^{*} \alpha_{\mathrm{T} 2 \mathrm{~g}}^{2}$ to describe the overall mixing of the nominal metal $3 \mathrm{~d}$ with the water orbitals. From Eq. (3) now one can derive an approximate relation for the average covalency for the $\left[\mathrm{Ni}_{2}\left(\mathrm{H}_{2} \mathrm{O}\right)_{6}\right]^{2+}$ complex:

$\left\langle\alpha^{2}\right\rangle=\frac{9 \frac{I_{L M C T}}{I_{L F}}}{9 \frac{I_{L M C T}}{I_{L F}}+10}$

Within the given approximations it is thus evident that the average $3 \mathrm{~d}$ covalency of the $\left[\mathrm{Ni}_{(}\left(\mathrm{H}_{2} \mathrm{O}\right)_{6}\right]^{2+}$ complex is given by the relative intensity of LMCT with respect to LF peaks in the $\mathrm{Ni}$ Ledge RIXS spectrum, without any additional theoretical simulations. The experimental requirement is that the LF and LMCT RIXS peaks can separated in the spectrum and their intensities determined.

The experimental $\mathrm{Ni} \mathrm{L}_{3}$-edge RIXS spectra of $\mathrm{Ni}^{2+}(\mathrm{aq})$ from Ref. [31] are displayed in Fig. 2(a), with clearly resolved LF and LMCT RIXS features. The $\mathrm{Ni}^{2+}$ (aq) L-edge X-ray absorption spectrum is well known $[7,25,31,37]$. The two LF peaks at energy transfers of 1.1 and $3.7 \mathrm{eV}$ can be assigned to a number of unresolved valence-excited LF states of the $\left(\mathrm{e}_{\mathrm{g}}^{*}, \mathrm{t}_{2 \mathrm{~g}}^{*}\right)^{8}$ configuration and the broad peak at $9.5 \mathrm{eV}$ corresponds to the LMCT final states of the $\left(e_{g}, t_{2 g}\right)^{9}\left(e_{g}^{*}, t_{2 g}^{*}\right)^{9}$ configuration [31]. In order to determine the relative intensities of the LF and LMCT peaks $\mathrm{I}_{\mathrm{LMCT}} / \mathrm{I}_{\mathrm{LF}}$ we integrate the RIXS intensities for incident photon energies of the $\mathrm{L}_{3}$ absorption edge (from 850 to $858 \mathrm{eV}$, Fig. 2(b)). $\mathrm{I}_{\mathrm{LMCT}} / \mathrm{I}_{\mathrm{LF}}$ is then determined from fitting this integrated spectrum (Fig. 2(b)) and we find $\mathrm{I}_{\mathrm{LMCT}} /$ $\mathrm{I}_{\mathrm{LF}}=0.065$. With Eq. (4) we determine the average ground-state covalency of the $\mathrm{Ni}$-water bond in the $\left[\mathrm{Ni}\left(\mathrm{H}_{2} \mathrm{O}\right)_{6}\right]^{2+}$ complex to 0.055 implying that the $\mathrm{Ni} 3 \mathrm{~d}$-dominated antibonding frontier orbitals have on average $5.5 \%$ of water-ligand character. The question occurs about how valid this concept is in general and for evaluating this we proceed to discussing the validity of the approximations introduced to derive Eq. (4).

First, Eq. (4) neglects multi-electron correlation (multiplet) effects, namely the $2 \mathrm{p}-3 \mathrm{~d}$ and $3 \mathrm{~d}-3 \mathrm{~d}$ Coulomb interactions and the $2 \mathrm{p}$ and $3 \mathrm{~d}$ spin-orbit interaction. We therefore assumed that the LF and LMCT features in the integrated RIXS spectrum of the complex can be assigned within the one-electron model by describing the corresponding RIXS final states with single determinants. However it is well-known that multiplet effects have to be accounted for when describing shapes and energies of L-edge RIXS of transition-metal complexes [32,38] and this is also evident from the multiplet structure in Fig. 2(a). It is thus somewhat surprising that their exclusion should yield a valid description. Second, the frozen-orbital approximation is applied in a sense that the inter-atomic core-hole screening is neglected and, therefore, the covalency $\alpha^{2}$ is the same for ground and core-excited states. The validity of these two approximations is addressed below and we find a posteriori that the approximations are justified for the case studied here. Note that we implicitly also employ the local approximation as routinely used to interpret XAS and RIXS data
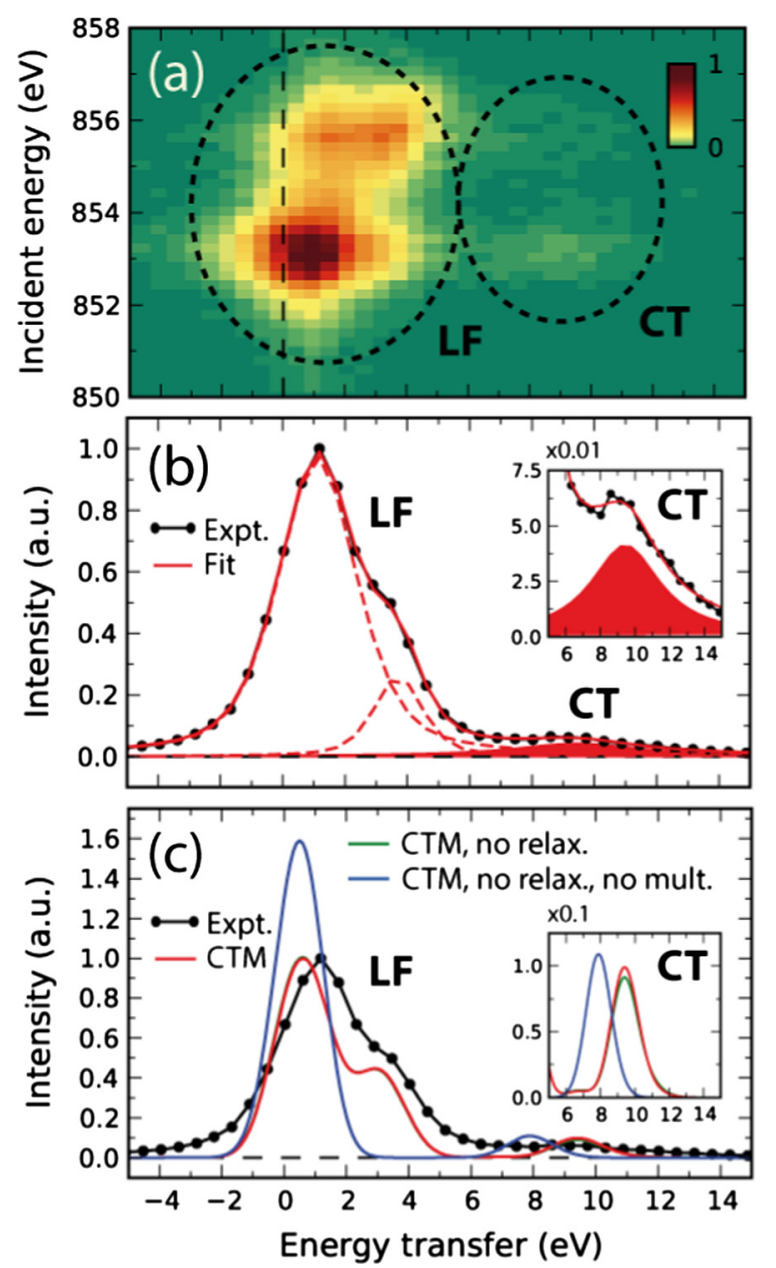

Fig. 2. (a) Experimental RIXS intensities of $\mathrm{Ni}^{2+}(\mathrm{aq})$ at the $\mathrm{Ni}_{3}$-edge encoded in color versus energy transfer and incident photon energy. Spectral features denoted with LF and CT correspond to ligand-field and ligand-to-metal charge-transfer excited states, respectively. (b) Filled circles with solid black line: Measured RIXS intensities versus energy transfer resulting from integrating all intensities in (a) over the displayed incident-photon-energy range (normalized to one at maximum). Solid red line: Fit of a sum of three pseudo-Voigt profiles (dashed red lines) to the measured data. The CT peak at an energy transfer of $9.5 \mathrm{eV}$ is highlighted by the filled red pseudo-Voigt profile and on an enlarged scale in the inset. According to the fit, the area of the CT peak divided by the total area of the LF features equals 0.065. (c) Solid red line: Result of a charge-transfer multiplet (CTM) calculation including electron correlation (multiplet) and core-hole relaxation effects. Solid green line: CTM calculation including multiplet but no core-hole effects (frozenorbital approximation, the line is barely visible as it is nearly identical to the red one, see the inset for differences). Solid blue line: CTM calculation without multiplet and core-hole relaxation effects (one-electron and frozen-orbital approximation). Filled circles with solid black line: Measured data, same as in (b). For details of the CTM calculations see text and Table 1. (For interpretation of the references to color in this figure legend, the reader is referred to the web version of this article.).

$[39,40]$ by discarding all non-local contributions to the RIXS intensities, i.e. contributions which arise from transitions of different atomic sites than the core-excited $\mathrm{Ni}$ site. In addition, we omit channel interference by assuming that independent absorption and emission steps can describe the RIXS process. Although interference between different resonant scattering channels is generally necessary to describe L-edge RIXS of 3d transition-metal ions [41], we have showed in Ref. [31] that the two step approximation is valid at the $\mathrm{Ni}$ L-edge of $\left[\mathrm{Ni}\left(\mathrm{H}_{2} \mathrm{O}\right)_{6}\right]^{2+}$ at both the $\mathrm{L}_{3}$ and $\mathrm{L}_{2}$ edges.

We address the one-electron and frozen-orbital approximations by performing a series of calculations using the well-established CTM model and the corresponding calculated spectra are 
compared to experiment in Fig. 2(c). The multiplet effects were included in a standard scheme where for the $2 \mathrm{p}-3 \mathrm{~d}$ and $3 \mathrm{~d}-3 \mathrm{~d}$ Coulomb interaction terms Hartree-Fock atomic values scaled by 0.9 were used and for the $2 \mathrm{p}$ and $3 \mathrm{~d}$ spin-orbit interactions the unscaled Hartree-Fock atomic values were used. In the CTM calculations, covalent interactions in $\left[\mathrm{Ni}_{(}\left(\mathrm{H}_{2} \mathrm{O}\right)_{6}\right]^{2+}$ with nominal $\mathrm{d}^{8}$ configuration are accounted for by an Anderson impurity model by coupling the states originating from $3 \mathrm{~d}^{8}$ and ligand (L)-to-metal LMCT $3 \mathrm{~d}^{9} \mathrm{~L}^{-1}$ configurations [42-44]. The extent of coupling is controlled by the charge transfer energy $\Delta_{\mathrm{g}}$ (difference between the average energies of the $3 \mathrm{~d}^{8}$ and $3 \mathrm{~d}^{9} \mathrm{~L}^{-1}$ multiplets) and by the mixing parameters $\mathrm{T}$ (non-diagonal elements of the Hamiltonian). For the calculations of the spectra in Fig. 2(c) the mixing parameters were set to $\mathrm{T}\left(\mathrm{E}_{\mathrm{g}}\right)=2.0 \mathrm{eV}$ and $\mathrm{T}\left(\mathrm{T}_{2 \mathrm{~g}}\right)=1.0 \mathrm{eV}$ and were kept the same also for the core-excited states. These values are typical for weakly covalent transition metal oxide compounds in local octahedral symmetry [32,45]. The RIXS final state energies are uniquely determined by the multiplet interactions in the $3 \mathrm{~d}^{8}$ configuration, the charge transfer energy $\Delta_{\mathrm{g}}$, and the mixing parameters $\mathrm{T}\left(\mathrm{E}_{\mathrm{g}}\right)$ and $\mathrm{T}\left(\mathrm{T}_{2 \mathrm{~g}}\right)$. The value for $\Delta_{\mathrm{g}}$ of $6.5 \mathrm{eV}$ was thus chosen to be the experimental separation of LF and LMCT energies. The core-hole relaxation effect can be simulated in the CTM model by using different values for the charge transfer energy $\Delta_{c}$ for the respective core-excited configurations $2 \mathrm{p}^{5} \mathrm{~d}^{9}$ and $2 \mathrm{p}^{5} \mathrm{~d}^{10} \mathrm{~L}^{-1}$. A difference of the charge transfer energies in the ground and core-excited states, $\Delta_{c}-\Delta_{g}$, is a resultant effect of the $2 \mathrm{p}$ core-hole potential $U_{\mathrm{pd}}$ and the valence electron repulsion $U_{\mathrm{dd}}$ caused by the addition of an extra electron to the $3 \mathrm{~d}$ manifold. It has been shown that in $3 \mathrm{~d}$ transition-metal compounds $U_{\mathrm{pd}}$ is larger than $\mathrm{U}_{\mathrm{dd}}$ by typically $1-2 \mathrm{eV}$ and, therefore, at the L-edge $-1 \mathrm{eV}>\Delta_{\mathrm{c}}-\Delta_{\mathrm{g}}>-2 \mathrm{eV}$ [32]. Given this parameter space we performed three CTM calculations, defined by the parameter sets given in Table 1 with the corresponding spectra displayed in Fig. 2(c). As for the experimental spectra, the calculated spectra in Fig. 2(c) result from integrating the calculated RIXS intensities for incident photon energies across the $L_{3}$ absorption edge. We display in Fig. 3 the calculated L-edge RIXS intensities versus energy transfer and incident photon energy.

For the CTM calculation with multiplet and core-hole relaxation effects ['CTM', Fig. 3(a) and red line in Fig. 2(c)] we find $\mathrm{I}_{\mathrm{LMCT}}$ / $\mathrm{I}_{\mathrm{LF}}=0.066$ in excellent agreement with experiment. Without core-hole relaxation this is smaller by $6 \%\left(\mathrm{I}_{\mathrm{LMCT}} / \mathrm{I}_{\mathrm{LF}}=0.062\right)$ and the spectral shapes of both the integrated RIXS spectrum ('CTM no relax.', green line in Fig. 2(c)) and of the full RIXS plane (Fig. 3 (b)) remain largely unchanged. The core-hole relaxation effect slightly increases $\mathrm{I}_{\mathrm{LMCT}} / \mathrm{I}_{\mathrm{LF}}$ (Fig. 2(c)) because lowering of $\Delta_{\mathrm{c}}$ with respect to $\Delta_{\mathrm{g}}$ increases covalent mixing between the core-excited configurations. We thus find that in the case of $\left[\mathrm{Ni}\left(\mathrm{H}_{2} \mathrm{O}\right)_{6}\right]^{2+}$ the core-hole relaxation has only a minor influence on $\mathrm{I}_{\mathrm{LMCT}} / \mathrm{I}_{\mathrm{LF}}$ (because $\Delta_{\mathrm{g}}>\mathrm{T}$ and $\Delta_{\mathrm{C}}>\mathrm{T}$ ) and as a consequence the core-hole induced change of covalency is small. We note that a so-called charge-transfer satellite peak in the X-ray absorption spectrum of $3 \mathrm{~d}$ transition metals was shown to be proportional to the change in mixing between $3 \mathrm{~d}^{\mathrm{n}}$ and $3 \mathrm{~d}^{\mathrm{n}+1} \mathrm{~L}^{-1}$ configurations [46]. Such a satellite peak is experimentally not detectable in the X-ray absorption spectrum of $\mathrm{Ni}^{2+}(\mathrm{aq})$, independently confirming that

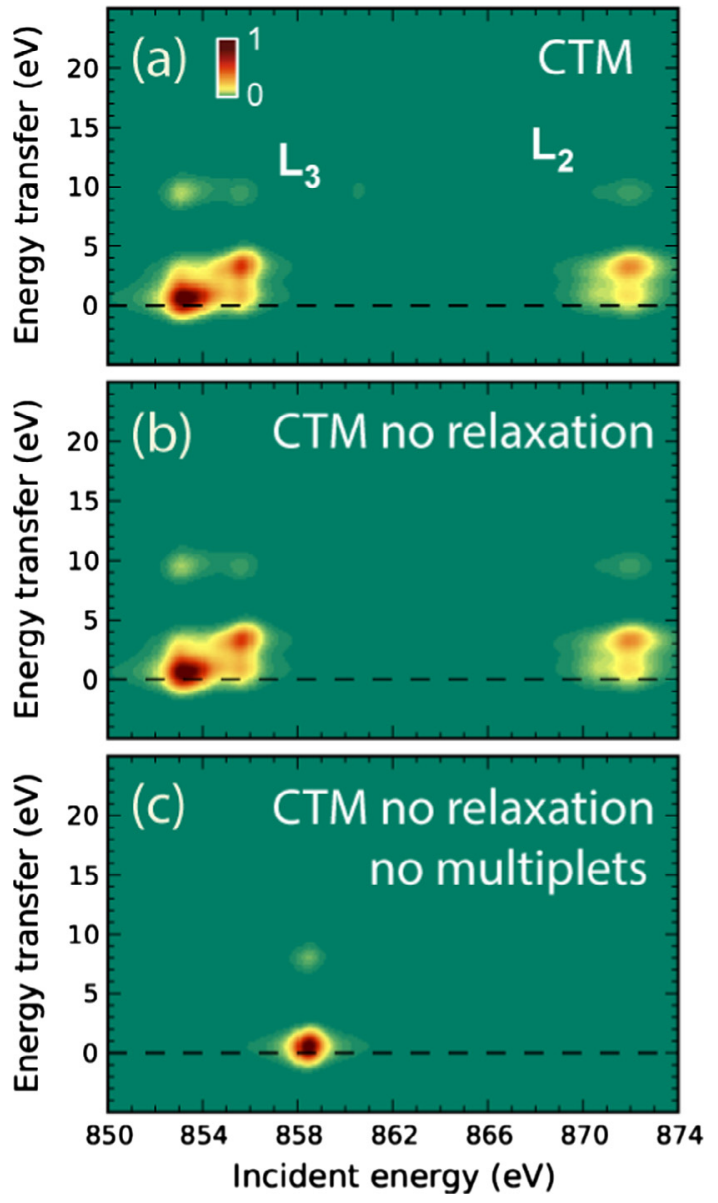

Fig. 3. Calculated $\mathrm{Ni}^{2+}(\mathrm{aq}) \mathrm{RIXS}$ intensities over the whole $\mathrm{Ni} \mathrm{L}_{2,3}$-edge. (a) CTM calculation including electron-correlation (multiplet) and core-hole relaxation effects ('CTM'), (b) CTM calculation including multiplet but no core-hole relaxation effects (frozen-orbital approximation, 'CTM no relaxation'). (c) CTM calculation without multiplet and core-hole relaxation effects (one-electron and frozen-orbital approximation, 'CTM no relaxation, no multiplets').

the core-hole relaxation influences the covalency in the coreexcited states to a minor degree only. The influence of core-hole relaxation on charge transfer could be further adjusted by lowering the mixing parameters in the core-excited configuration to model the effect of radial contraction of the Ni orbitals due to positively charged core-hole [46]. Contrary to the effect of lowering $\Delta_{c}$ respect to $\Delta_{\mathrm{g}}$, this would decrease the $\mathrm{I}_{\mathrm{LMCT}} / \mathrm{I}_{\mathrm{LF}}$. We have left here the mixing parameters unchanged because the CTM calculation with multiplet and core-hole relaxation effects already reproduces very well the experimental observable $\mathrm{I}_{\mathrm{LMCT}} / \mathrm{I}_{\mathrm{LF}}$. The measured shape of the LMCT feature is not well reproduced in the CTM calculation. This can be explained by the various ligand-orbital energies being approximated by a single parameter $\Delta_{\mathrm{g}}$. In the third ("CTM, no relax., only 2p SO') and fourth calculation ['CTM no relx., no mult.', Fig. 3(c) and blue line in Fig. 2(c)] both multiplet and

Table 1

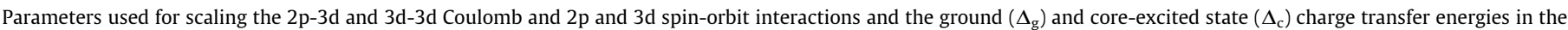

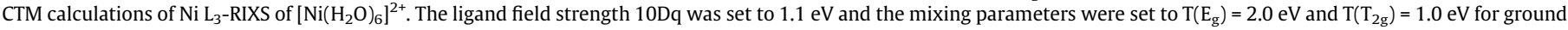
and core-excited states. See text for more details.

\begin{tabular}{|c|c|c|c|c|c|}
\hline & Scaling of Coulomb interactions & Scaling of $2 p$ spin-orbit interactions & Scaling of $3 \mathrm{~d}$ spin-orbit interactions & $\Delta_{\mathrm{g}}$ & $\Delta_{\mathrm{c}}$ \\
\hline CTM & 0.9 & 1 & 1 & 6.5 & 5.5 \\
\hline CTM, no relax. & 0.9 & 1 & 1 & 6.5 & 6.5 \\
\hline CTM, no relax., only $2 \mathrm{p}$ SO & 0 & 1 & 0 & 6.5 & 6.5 \\
\hline CTM, no relax., no mult. & 0 & 0 & 0 & 6.5 & 6.5 \\
\hline
\end{tabular}


Table 2

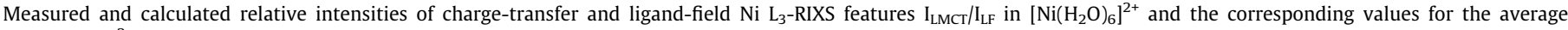
covalency $\left\langle\alpha^{2}\right\rangle$ following from Eq. (4).

\begin{tabular}{|c|c|c|c|c|c|c|}
\hline & \multicolumn{3}{|c|}{$\mathrm{I}_{\mathrm{LMCT}} / \mathrm{I}_{\mathrm{LF}}$} & \multicolumn{3}{|l|}{$\left\langle\alpha^{2}\right\rangle$} \\
\hline & $\mathrm{L}_{3}$ & $\mathrm{~L}_{2}$ & $\mathrm{~L}_{2,3}$ & $\mathrm{~L}_{3}$ & $\mathrm{~L}_{2}$ & $\mathrm{~L}_{2,3}$ \\
\hline Expt. & 0.065 & - & - & 0.055 & - & - \\
\hline CTM & 0.066 & 0.077 & 0.074 & 0.056 & 0.065 & 0.062 \\
\hline CTM, no relax. & 0.062 & 0.075 & 0.068 & 0.053 & 0.063 & 0.058 \\
\hline CTM, no relax., only 2p SO & 0.059 & 0.062 & 0.060 & 0.050 & 0.053 & 0.051 \\
\hline CTM, no relax., no mult. & - & - & 0.066 & - & - & 0.056 \\
\hline
\end{tabular}

core-hole relaxation effects were turned off. The fourth calculation is thus performed at the same level of approximations as used for deriving Eqs. (3) and (4). As expected, the shape of the calculated RIXS plane changes drastically and has no resemblance with the full calculation including all relevant effects (Fig. 3). Interestingly, however, the calculated integrated RIXS spectrum still shows acceptable agreement with the measured integrated RIXS spectrum (Fig. 2). Even more notably, the calculated $\mathrm{I}_{\mathrm{LMCT}} / \mathrm{I}_{\mathrm{LF}}$ ratio of 0.066 does not change compared to the calculation including both multiplet and core-hole relaxation effects. It is likely coincidental that the multiplet effects so exactly balance out the core-hole relaxation effects in the current case. This is suggested by the comparison with calculated results at the L2 edge which show systematically higher $\mathrm{I}_{\mathrm{LMCT}} / \mathrm{I}_{\mathrm{LF}}$ ratios (Table 2 ). Nevertheless, it is clear that the multiplet effects have also only a small influence on the $\mathrm{I}_{\mathrm{LMCT}} /$ $\mathrm{I}_{\mathrm{LF}}$ ratio. Our calculations therefore demonstrate that the oneelectron approximation and the frozen-orbital approximation used to derive Eqs. (3) and (4) are well justified in case of $\left.\left[\mathrm{Ni}^{(} \mathrm{H}_{2} \mathrm{O}\right)_{6}\right]^{2+}$. All calculated $\mathrm{I}_{\mathrm{LMCT}} / \mathrm{I}_{\mathrm{LF}}$ ratios and the corresponding average covalencies $\left\langle\alpha^{2}\right\rangle$ with Eq. (4) are compared to experiment in Table 2. It will be interesting in future studies to compare the experimentally derived values for the $\mathrm{Ni}^{2+}-\mathrm{H}_{2} \mathrm{O}$ covalency with calculated values. In addition, the concept presented here could be further tested on other $3 \mathrm{~d}$ transition metal ions in water thereby systematizing how multiplet and core-hole relaxation effects influence the RIXS spectra and the derived covalency.

\section{Summary and conclusions}

Summarizing, we showed that the relative intensity of LMCT and LF spectral features in the Ni L-edge resonant inelastic X-ray scattering spectrum of the hexaaqua complex $\left.\left[\mathrm{Ni}^{(} \mathrm{H}_{2} \mathrm{O}\right)_{6}\right]^{2+}$ is dominantly determined by the ground state covalent mixing between the $\mathrm{Ni} 3 \mathrm{~d}$ orbitals and the water-ligand orbitals. Based on a derived relationship of this intensity ratio and the ground-state covalency accounting for the occupation of the respective molecular orbitals allows us to derive the average covalent contribution to the $\mathrm{Ni}^{2+}$ solute and water solvent interactions. We arrive at 0.055 meaning that the nominal $\mathrm{Ni} 3 \mathrm{~d}$ antibonding molecular orbitals have an average of $5.5 \%$ of ligand $\mathrm{H}_{2} \mathrm{O} 1 \mathrm{~b}_{1}$ and $3 \mathrm{a}_{1}$ character. This represents a stringent test for modern quantum chemistry approaches. We consider it possible that the conclusions drawn here are valid not only for weakly covalent $\mathrm{Ni}^{2+}$ complexes, but potentially also for complexes with different metals, oxidations states and higher covalency.

\section{Acknowledgments}

K.K. acknowledges Thomas Kroll for fruitful discussions. M.O. acknowledges financial support from the Swedish Research Council and Carl Tryggers foundation. We gratefully acknowledge the continuous support by the BESSY II staff and we thank HZB for the allocation of synchrotron radiation beamtime. Portions of the presented research were funded by the Helmholtz Virtual Institute 'Dynamic Pathways in Multidimensional Landscapes'.

\section{References}

[1] G.N. Lewis, The atom and the molecule, J. Am. Chem. Soc. 38 (1916) 762-785

[2] L.A. Näslund, M. Cavalleri, H. Ogasawara, A. Nilsson, L.G.M. Pettersson, P. Wernet, et al., Direct evidence of orbital mixing between water and solvated transition-metal ions: an oxygen 1s XAS and DFT study of aqueous systems, J. Phys. Chem. A 107 (2003) 6869-6876, http://dx.doi.org/10.1021/jp034296h.

[3] A. Werner, Beitrag zur Konstitution anorganisher Verbindungen, Z. Anorg. Chem. 3 (1893) 267-330.

[4] B.N. Figgis, M.A. Hitchman, Ligand Field Theory and Its Applications, WileyVCH, 2000.

[5] R. Åkesson, L.G.M. Pettersson, M. Sandstrom, U. Wahlgren, Ligand-field effects in the hydrated divalent and trivalent metal-ions of the first and 2nd transition periods, J. Am. Chem. Soc. 116 (1994) 8691-8704.

[6] S. Bonhommeau, N. Ottosson, W. Pokapanich, S. Svensson, W. Eberhardt, O. Bjorneholm, et al., Solvent effect of alcohols at the L-edge of iron in solution: Xray absorption and multiplet calculations, J. Phys. Chem. B 112 (2008) 12571 12574, http://dx.doi.org/10.1021/jp8071266.

[7] E.F. Aziz, The solvation of ions and molecules probed via soft X-ray spectroscopies, J. Electron Spectrosc. Relat. Phenom. 177 (2010) 168-180, http://dx.doi.org/10.1016/j.elspec.2010.02.009.

[8] P. Wernet, K. Kunnus, S. Schreck, W. Quevedo, R. Kurian, S. Techert, et al. Dissecting local atomic and intermolecular interactions of transition-metal ions in solution with selective X-ray spectroscopy, J. Phys. Chem. Lett. 3 (2012) 3448-3453.

[9] K. Atak, S.I. Bokarev, M. Gotz, R. Golnak, K.M. Lange, N. Engel, et al., Nature of the chemical bond of aqueous $\mathrm{Fe}^{2+}$ probed by soft X-ray spectroscopies and ab initio calculations, J. Phys. Chem. B 117 (2013), http://dx.doi.org/10.1021/ jp408212u.

[10] B. Winter, M. Faubel, Photoemission from liquid aqueous solutions, Chem. Rev. 106 (2006) 1176-1211, http://dx.doi.org/10.1021/cr040381p.

[11] S. Thürmer, R. Seidel, W. Eberhardt, S.E. Bradforth, B. Winter, Ultrafast hybridization screening in $\mathrm{Fe}^{3+}$ aqueous solution, J. Am. Chem. Soc. 133 (2011) 12528-12535, http://dx.doi.org/10.1021/ja200268b.

[12] R. Golnak, S.I. Bokarev, R. Seidel, J. Xiao, G. Grell, K. Atak, et al., Joint analysis of radiative and non-radiative electronic relaxation upon X-ray irradiation of transition metal aqueous solutions, Sci. Rep. 6 (2016) 24659, http://dx.doi.org/ $10.1038 /$ srep24659.

[13] U. Bergmann, C.R. Horne, TJ. Collins, J.M. Workman, S.P. Cramer, Chemical dependence of interatomic X-ray transition energies and intensities - a study of $\mathrm{Mn} \mathrm{K} \beta^{\prime \prime}$ and $K \beta_{2,5}$ spectra, Chem. Phys. Lett. 302 (1999) 119-124, http://dx. doi.org/10.1016/S0009-2614(99)00095-0.

[14] N. Lee, T. Petrenko, U. Bergmann, F. Neese, S. DeBeer, Probing valence orbital composition with iron K $\beta$ X-ray emission spectroscopy, J. Am. Chem. Soc. 132 (2010) 9715-9727, http://dx.doi.org/10.1021/ja101281e.

[15] D.A. Meyer, X. Zhang, U. Bergmann, K.J. Gaffney, Characterization of charge transfer excitations in hexacyanomanganate(III) with Mn K-edge resonant inelastic X-ray scattering, J. Chem. Phys. 132 (2010) 134502, http://dx.doi.org/ 10.1063/1.3367958.

[16] C.J. Pollock, M.U. Delgado-Jaime, M. Atanasov, F. Neese, S. DeBeer, K $\beta$ mainline $\mathrm{X}$-ray emission spectroscopy as an experimental probe of metal-ligand covalency, J. Am. Chem. Soc. 136 (2014) 9453-9463, http://dx.doi.org/ 10.1021/ja504182n.

[17] M.A. Beckwith, M. Roemelt, M.-N. Collomb, C. DuBoc, T.-C. Weng, U. Bergmann, et al., Manganese $\mathrm{K} \beta \mathrm{X}$-ray emission spectroscopy as a probe of metal-ligand interactions, Inorg. Chem. 50 (2011) 8397-8409, http://dx.doi.org/10.1021/ ic200970t.

[18] M. Lundberg, T. Kroll, S. Debeer, U. Bergmann, S.A. Wilson, P. Glatzel, et al., Metal-ligand covalency of iron complexes from high-resolution resonant inelastic X-ray scattering. J. Am. Chem. Soc. 135 (2013) 17121-17134.

[19] Erik C. Wasinger, Frank M.F. de Groot, Britt Hedman, Keith O. Hodgson, Edward I. Solomon, L-edge X-ray absorption spectroscopy of non-heme iron sites: experimental determination of differential orbital covalency, 2003. http:// dx.doi.org/10.1021/JA034634S.

[20] R.K. Hocking, E.C. Wasinger, F.M.F. de Groot, K.O. Hodgson, B. Hedman, E.I. Solomon, Fe L-edge XAS studies of $\mathrm{K}_{4}\left[\mathrm{Fe}(\mathrm{CN})_{6}\right]$ and $\mathrm{K}_{3}\left[\mathrm{Fe}(\mathrm{CN})_{6}\right]$ : a direct probe 
of back-bonding, J. Am. Chem. Soc. 128 (2006) 10442-10451, http://dx.doi.org/ 10.1021/ja061802i.

[21] E. Suljoti, R. Garcia-Diez, S.I. Bokarev, K.M. Lange, R. Schoch, B. Dierker, et al., Direct observation of molecular orbital mixing in a solvated organometallic complex, Angew. Chem. - Int. Ed. 52 (2013) 9841-9844, http://dx.doi.org/ 10.1002/anie.201303310.

[22] N. Engel, S.I. Bokarev, E. Suljoti, R. Garcia-Diez, K.M. Lange, K. Atak, et al., Chemical bonding in aqueous ferrocyanide: experimental and theoretical $\mathrm{X}$ ray spectroscopic study, J. Phys. Chem. B 118 (2014) 1555-1563, http://dx.doi. org/10.1021/jp411782y.

[23] T. Kroll, E.I. Solomon, F.M.F. de Groot, Final-state projection method in chargetransfer multiplet calculations: an analysis of Ti L-edge absorption spectra, J Phys. Chem. B 119 (2015) 13852-13858, http://dx.doi.org/10.1021/acs. jpcb.5b04133.

[24] K. Kunnus, W. Zhang, M.G. Delcey, R.V. Pinjari, P.S. Miedema, S. Schreck, et al., Viewing the valence electronic structure of ferric and ferrous hexacyanide in solution from the Fe and cyanide, Perspectives (2016).

[25] I. Josefsson, K. Kunnus, S. Schreck, A. Föhlisch, F. De Groot, P. Wernet, et al., Ab initio calculations of X-ray spectra: Atomic multiplet and molecular orbital effects in a multiconfigurational scf approach to the L-edge spectra of transition metal complexes, J. Phys. Chem. Lett. 3 (2012) 3565-3570, http:// dx.doi.org/10.1021/jz301479j.

[26] M. Roemelt, D. Maganas, S. DeBeer, F. Neese, A combined DFT and restricted open-shell configuration interaction method including spin-orbit coupling: application to transition metal L-edge X-ray absorption spectroscopy, J. Chem. Phys. 138 (2013) 204101.

[27] S.I. Bokarev, M. Dantz, E. Suljoti, O. Kühn, E.F. Aziz, State-dependent electron delocalization dynamics at the solute-solvent interface: soft-X-ray absorption spectroscopy and Ab initio calculations, Phys. Rev. Lett. 111 (2013) 83002, http://dx.doi.org/10.1103/PhysRevLett. 111.083002.

[28] R.V. Pinjari, M.G. Delcey, M. Guo, M. Odelius, M. Lundberg, Restricted active space calculations of L-edge X-ray absorption spectra: from molecular orbitals to multiplet states, J. Chem. Phys. 141 (2014) 124116, http://dx.doi.org/ 10.1063/1.4896373.

[29] M. Preuße, S.I. Bokarev, S.G. Aziz, O. Kühn, Towards an ab initio theory for metal L-edge soft X-ray spectroscopy of molecular aggregates, Struct. Dyn. 3 (2016) 62601, http://dx.doi.org/10.1063/1.4961953.

[30] E.I. Solomon, B. Hedman, K.O. Hodgson, A. Dey, R.K. Szilagyi, Ligand K-edge Xray absorption spectroscopy: covalency of ligand-metal bonds, Coord. Chem. Rev. 249 (2005) 97-129, http://dx.doi.org/10.1016/j.ccr.2004.03.020.

[31] K. Kunnus, I. Josefsson, S. Schreck, W. Quevedo, P.S. Miedema, S. Techert, et al., From ligand fields to molecular orbitals: probing the local valence electronic structure of $\mathrm{Ni}^{2+}$ in aqueous solution with resonant inelastic X-ray scattering, J. Phys. Chem. B 117 (2013) 16512-16521, http://dx.doi.org/10.1021/jp4100813.

[32] F.M.F. de Groot, A. Kotani, Core Level Spectroscopy of Solids, CRC Press, Boca Raton, 2008.
[33] B.T. Thole, G. van der Laan, J.C. Fuggle, G.A. Sawatzky, R.C. Karnatak, J.-M. Esteva, 3d $x$-ray-absorption lines and the $3 \mathrm{~d}^{9} 4 \mathrm{f}^{\mathrm{f}+1}$ multiplets of the lanthanides, Phys. Rev. B 32 (1985) 5107-5118, http://dx.doi.org/10.1103/ PhysRevB.32.5107.

[34] F.M.F. de Groot, J.C. Fuggle, B.T. Thole, G.A. Sawatzky, 2p X-ray absorption of 3d transition-metal compounds - an atomic multiplet description including the crystal-field, Phys. Rev. B 42 (1990) 5459-5468, http://dx.doi.org/10.1103/ PhysRevB.42.5459.

[35] R.J. Green, D.A. Zatsepin, D.J. St, E.Z. Onge, N.V. Kurmaev, A.F..Zatsepin. Gavrilov, et al., Electronic band gap reduction and intense luminescence in Co and $\mathrm{Mn}$ ion-implanted $\mathrm{SiO}_{2}$, J. Appl. Phys. 115 (2014) 103708, http://dx.doi. org/10.1063/1.4868297.

[36] B. Kallies, R. Meier, Electronic structure of $3 \mathrm{~d} \mathrm{M}\left(\mathrm{H}_{2} \mathrm{O}\right)_{6}^{3+}$ ions from Sc-III to FeIII: a quantum mechanical study based on DFT computations and natural bond orbital analyses, Inorg. Chem. 40 (2001) 3101-3112, http://dx.doi.org/ 10.1021/ic001258t.

[37] R. Seidel, S. Thürmer, B. Winter, Photoelectron spectroscopy meets aqueous solution: studies from a vacuum liquid microjet, J. Phys. Chem. Lett. 2 (2011) 633-641, http://dx.doi.org/10.1021/jz101636y.

[38] F.M.F. de Groot, Multiplet effects in X-ray spectroscopy, Coord. Chem. Rev. 249 (2005) 31-63, http://dx.doi.org/10.1016/j.ccr.2004.03.018.

[39] J. Stöhr, NEXAFS Spectroscopy, Springer, Berlin, 1992.

[40] R. Manne, Molecular orbital interpretation of x-ray emission spectra - simple hydrocarbons and carbon oxides, J. Chem. Phys. 52 (1970) 5733-5739, http:// dx.doi.org/10.1063/1.1672852.

[41] R. Kurian, K. Kunnus, P. Wernet, S.M. Butorin, P. Glatzel, F.M.F. de Groot, Intrinsic deviations in fluorescence yield detected $\mathrm{x}$-ray absorption spectroscopy: the case of the transition metal $\mathrm{L}_{2,3}$ edges, J. Phys. Condens. Matter 24 (2012) 452201.

[42] A. Fujimori, F. Minami, Valence-band photoemission and optical absorption in nickel compounds, Phys. Rev. B 30 (1984) 957-971, http://dx.doi.org/10.1103/ PhysRevB.30.957.

[43] J. Zaanen, C. Westra, G.A. Sawatzky, Determination of the electronic-structure of transition-metal compounds - 2p x-ray photoemission spectroscopy of the nickel dihalides, Phys. Rev. B 33 (1986) 8060-8073, http://dx.doi.org/10.1103/ PhysRevB.33.8060.

[44] A. Kotani, H. Ogasawara, Theory of core-level spectroscopy of rare-earth oxides, J. Electron Spectrosc. Relat. Phenom. 60 (1992) 257-299, http://dx.doi. org/10.1016/0368-2048(92)80024-3.

[45] L.F. Mattheiss, Electronic structure of the 3d transition-metal monoxides. I. Energy-band results, Phys. Rev. B 5 (1972) 290-306, http://dx.doi.org/10.1103/ PhysRevB.5.290.

[46] F.M.F. de Groot, X-ray-absorption and dichroism of transition-metals and their compounds, J. Electron Spectrosc. Relat. Phenom. 67 (1994) 529-622, http:// dx.doi.org/10.1016/0368-2048(93)02041-j. 